\title{
Rapid one-step detection of SARS-CoV-2 RNA
}

\author{
A one-pot fluorescence-based assay detects SARS-CoV-2 RNA in under an hour with high sensitivity and sequence \\ specificity.
}

\section{Arun Richard Chandrasekaran, Lifeng Zhou and Ken Halvorsen}

T he large-scale testing and monitoring of infections by severe acute respiratory syndrome coronavirus 2 (SARS-CoV-2) is central to controlling outbreaks of coronavirus disease 2019 (COVID-19) ${ }^{1}$. The gold-standard method for viral detection in clinical samples is real-time polymerase chain reaction (rtPCR), which involves nucleic acid amplification and its monitoring via fluorescence. Because SARS-CoV-2 mainly exists in the respiratory tract, samples for analysis by rtPCR are typically collected from nasal or oral swabs, and then processed for the extraction of viral RNA. However, testing with $\mathrm{rtPCR}$ requires expensive equipment available mostly in specialized laboratories and the use of reagents ${ }^{2}$ whose availability has been affected by disruptions in global supply chains. Hence, testing facilities have been slow in ramping up their capabilities and have not always kept pace with demand, resulting in long turnaround times (many days) that have undermined the utility of the tests.

Difficulties in carrying out population-wide testing during a pandemic call for the development of a diverse range of deployable testing assays. The design and evaluation of diagnostic tests has shifted from a narrow focus on analytic sensitivity and specificity towards cost, turnaround time and ease of use by non-specialists operating outside laboratory settings ${ }^{3}$ (such as workers in public transport hubs, social care and schools). A point-of-care test that is sufficiently inexpensive and fast enables healthcare decisions, even if the test cannot meet best-in-class benchmark analytical limits of detection ${ }^{4,5}$. In fact, for surveillance studies of viral disease, the frequency and speed of testing may be more practically relevant than test sensitivity ${ }^{6}$. For COVID-19, this is supported by the fact that high viral loads are most likely to be related to infectivity ${ }^{7}$, and that they correlate with COVID-19 severity . $^{8}$

The challenges faced by traditional in vitro diagnostic techniques, and the need for more frequent testing, have accelerated the development of alternative COVID-19
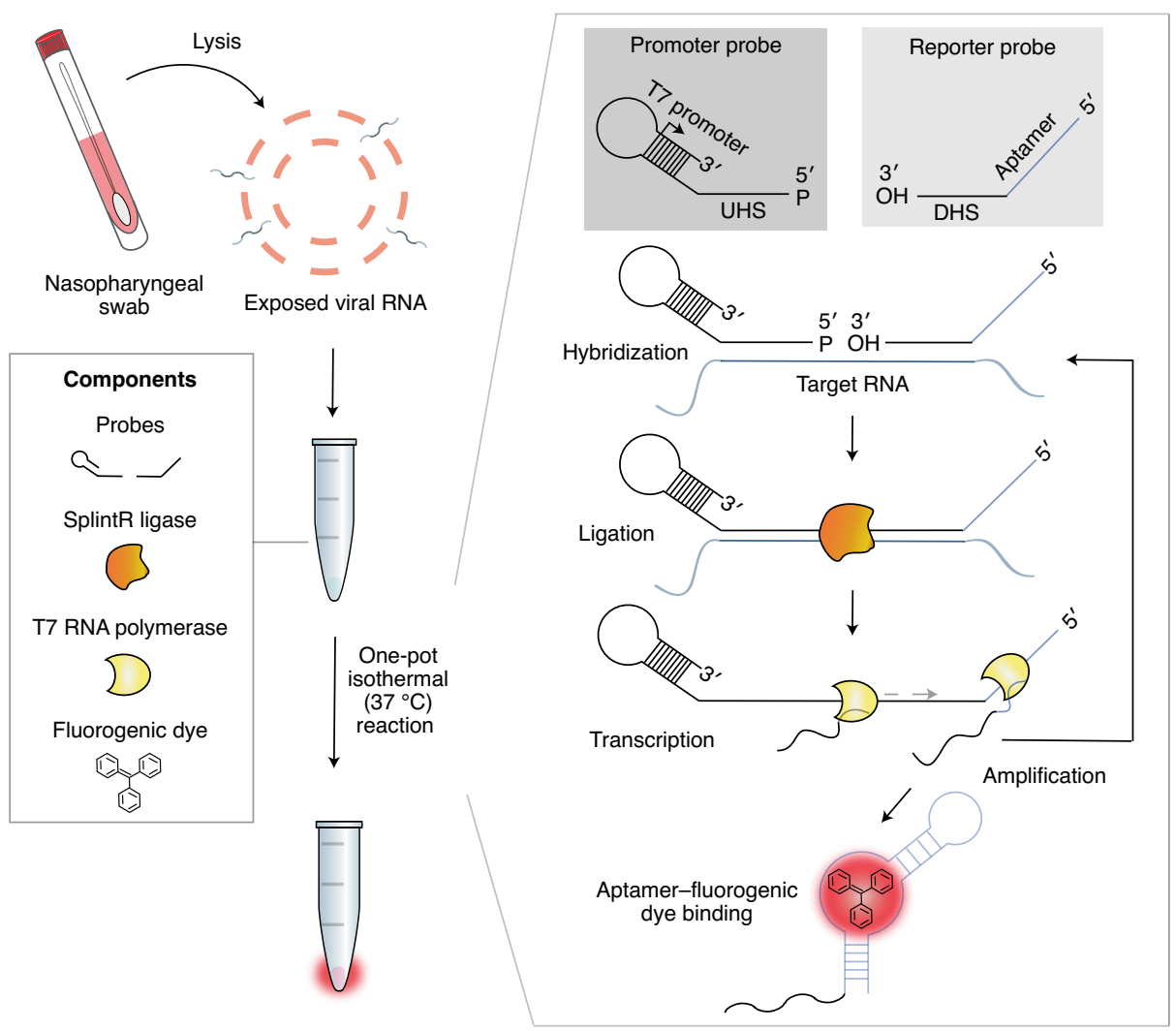

Fig. 1 | One-pot isothermal assay for the rapid detection of viral RNA. The assay workflow involves the lysis of samples to release RNA, and the addition, in a single pot, of DNA probes, SplintR ligase, T7 RNA polymerase and a fluorogenic dye at $37^{\circ} \mathrm{C}$. The single-pot reaction involves the hybridization and ligation of the promoter and reporter probes with the viral RNA, and the polymerase-mediated transcription of the dye-binding aptamer. UHS, upstream hybridization sequence; DHS, downstream hybridization sequence. Figure adapted with permission from ref. ${ }^{9}$, Springer Nature Ltd.

testing strategies ${ }^{1}$. Reporting in Nature Biomedical Engineering, Jeong Wook Lee, Gyoo Yeol Jung and colleagues now describe a one-pot isothermal assay for the detection of SARS-CoV-2 RNA in clinical samples in less than one hour'. The approach builds on previous techniques that use splint ligation ${ }^{10}$ - whereby two DNA molecules are ligated, followed by the transcription of an RNA aptamer that binds to a fluorescent dye $\mathrm{e}^{11}$.

Lee, Jung and co-authors' assay involves four core components: two DNA probes, a SplintR ligase (which ligates two single-stranded DNA molecules, splinted by a complementary RNA strand), a T7 RNA polymerase and a fluorogenic dye (Fig. 1). One of the DNA probes is a stem-loop hairpin corresponding to the $\mathrm{T} 7$ promoter region, and contains a single-stranded segment that is complementary to part of the target RNA region. The other DNA probe contains a segment that binds to the other half of the target RNA region, and a template sequence 
for a dye-binding RNA aptamer. Following splinting and ligation steps, the T7 RNA polymerase transcribes the ligated DNA template to synthesize the RNA aptamer sequence that binds to the fluorogenic dye. The high efficiency of ligation and RNA transcription also serves to amplify the target RNA in the same reaction, producing enough product to capture the fluorogenic dye and to emit fluorescence. The authors used rtPCR and electrophoresis to verify the ligation of the two DNA probes and the transcription of the RNA, and further optimized the reaction temperature and buffer conditions so that the assay can be completed within 1 hour of incubation at $37^{\circ} \mathrm{C}$ with a 0.1 aM limit of detection.

The assay can be adapted for a broad range of target sequences by redesigning the sequences of the two DNA probes. Lee, Jung and co-authors reprogrammed the assay to detect RNA from other pathogens, in particular the Middle East respiratory syndrome-related coronavirus, the influenza A virus, Staphylococcus aureus, Vibrio vulnificus and Escherichia coli. The authors also show that the assay can detect two pathogens simultaneously by using two different types of RNA-aptamerfluorescent-dye pairs. Notably, the assay does not require RNA extraction; RNA can be released for detection from whole bacterial cells in human serum and urine simply by lysing them (by heating at $95^{\circ} \mathrm{C}$ for 2 minutes). In fact, for the detection of SARS-CoV-2 RNA in clinical samples, the authors used samples from nasopharyngeal swabs, and included a thermal or chemical lysis step to release the RNA in the viral lysate. For 40 clinical nasopharyngeal samples that had been tested by rtPCR, the assay led to positive and negative predictive values of $95 \%$ and $100 \%$, respectively. In most cases, detection was completed in 35-55 minutes, from sample preparation to fluorescence readout. The authors demonstrate the specificity of the assay by discriminating between seven different coronavirus variants with high sequence homology.

The programmability of Lee, Jung and colleagues' assay facilitates its adaptation for the detection of a wide range of viral and bacterial pathogens. The one-pot reaction provides an advantage over the typical multistep sample-preparation and amplification processes of most diagnostic assays, and the short turnaround time should help facilitate the development of the assay for use at the point of care. However, progressing from a proof-of-concept demonstration to use in a clinical laboratory or in a point-of-care setting involves a number of challenges. Adapting assays for clinical use involves scaled-up manufacturing under current good-manufacturing-practice guidelines and distribution of the test materials and reagents, as well as the integration of the assay into workflows for sample collection, storage and processing. For point-of-care testing, additional requirements such as cold storage for the enzyme cocktail and laboratory equipment for the assay's incubation and readout steps present further hurdles to overcome. These are solvable challenges, likely to be best suited for a company or for an academic-corporate partnership with commercialization as the end goal. The simplicity, adaptability and speed of Lee, Jung and colleagues' assay should be advantageous, and may help to diversify the expanding suite of diagnostic tests for SARS-CoV-2 (refs. ${ }^{12-15}$ ) and for emerging infectious pathogens.

Arun Richard Chandrasekaran, Lifeng Zhou and Ken Halvorsen (1D $\square$

The RNA Institute, University at Albany, State University of New York, Albany, NY, USA.

$凶_{e-m a i l: k h a l v o r s e n @ a l b a n y . e d u}$

Published online: 8 December 2020 https://doi.org/10.1038/s41551-020-00663-z

References

1. Udugama, B. et al. ACS Nano 14, 3822-3835 (2020).

2. Esbin, M. N. et al. RNA 26, 771-783 (2020).

3. Mina, M. J., Parker, R. \& Larremore, D. B. N. Engl. J. Med https://doi.org/10.1056/NEJMp2025631 (2020).

4. Olena, A. Toward COVID-19 testing any time, anywhere. The Scientist https://go.nature.com/32ZkXlY (2 September 2020).

5. Schnipper, J. L. \& Sax, P. E. Even imperfect Covid-19 tests can help control the pandemic. STAT https://go.nature.com/2IRhS0i (20 August 2020).

6. Larremore, D. B. et al. Sci. Adv. https://doi.org/10.1126/sciadv. abd5393 (2020).

7. He, X. et al. Nat. Med. 26, 672-675 (2020)

8. Pujadas, E. et al. Lancet Respir. Med. 8, e70 (2020)

9. Woo, C. H., Jang, S., Shin, G., Jung, G. Y. \& Lee, J. W. Nat. Biomed. Eng. https://doi.org/10.1038/s41551-020-00617-5 (2020).

10. Jin, J. et al. Nucleic Acids Res. 44, el16 (2016).

11. Ying, Z.-M. et al. Chem. Commun. 54, 3010-3013 (2018).

12. Joung, J. et al. N. Engl. J. Med. 383, 1492-1494 (2020).

13. Ganguli, A. et al. Proc. Natl Acad. Sci. USA 117, 22727-22735 (2020)

14. Broughton, J. P. et al. Nat. Biotechnol. 38, 870-874 (2020).

15. Zhou, L. et al. Sci. Adv. 6, eabc6246 (2020).

\section{Competing interests}

The authors are actively developing, and hold patents and patent applications, on viral-detection technology. 